\title{
Pesticide exposure and cancer: an integrative literature review
}

\author{
Exposição a agrotóxicos e câncer: uma revisão integrativa da \\ literatura
}

Thaís Bremm Pluth', Lucas Adalberto Geraldi Zanini2,3, lara Denise Endruweit Battisti ${ }^{1}$

DOI: $10.1590 / 0103-1104201912220$

\begin{abstract}
We conducted an integrative literature review of published studies on pesticide and cancer exposure, focusing on farmers, rural population, pesticide applicators, and rural workers. The Medline/PubMed was used as searching database. After the retrieval, 74 articles were selected according to pre-established criteria, which design involved 39 case-controls, 32 cohorts, 2 ecological ones, and 1 cross-sectional. Among them, 64 studies showed associations between pesticides and cancer while 10 did not find any significant association. The studies found 53 different types of pesticides significantly associated with at least one type of cancer and 19 different types of cancers linked to at least one type of pesticide. Although few studies presented contradictory results, the sole fact of being a farmer or living near crops or high agricultural areas have also been used as a proxy for pesticide exposure and significantly associated with higher cancer risk. The literature well illustrates the case of prostate cancer, Non-Hodgkin lymphoma, leukemia, multiple myeloma, bladder and colon cancers. Studies are recommended to further investigate the relationship between pesticide and neoplasm of testis, breast, esophagus, kidney, thyroid, lip, head and neck, and bone.
\end{abstract}

KEYWORDS Neoplasms. Agrochemicals. Occupational diseases. Review.

RESUMO Trata-se de revisão integrativa da literatura sobre estudos publicados em relação à exposição a agrotóxicos e câncer, com foco em agricultores, população rural, aplicadores de agrotóxicos e trabalhadores rurais. A busca dos artigos foi realizada por meio do banco de dados Medline/ PubMed. Após a triagem, 74 artigos foram selecionados de acordo com critérios pré-estabelecidos, sendo 39 caso-controle, 32 coortes, dois ecológicos e um transversal. Desses, 64 estudos mostraram associação entre agrotóxicos e câncer, enquanto dez não encontraram associação significativa. Nesses 64, 53 diferentes tipos de agrotóxicos foram significativamente associados com pelo menos um tipo de câncer e, inversamente, 19 diferentes tipos de câncer foram associados a pelo menos um tipo de agrotóxico. Embora alguns estudos tenham apresentado resultados contraditórios, ser

1 Universidade Federal da Fronteira Sul (UFFS) Cerro Largo (RS), Brazil. thaisbremm@hotmail.com

2 Hospital de Caridade de ljuí (HCl) - ljuí (RS), Brazil.

3 Universidade Regional do Noroeste do Estado do Rio Grande do Sul (Unijuí)- ljuí (RS), Brazil. um agricultor ou morar perto de plantações ou de áreas densamente agrícolas também tem sido motivo para representar a exposição a agrotóxicos e considerado significativamente associado a um maior risco de câncer. A literatura ilustra bem o câncer de próstata, linfoma não-Hodgkin, leucemia, mieloma múltiplo, bexiga e câncer de cólon. Recomendam-se estudos que investiguem mais a relação entre agrotóxicos e neoplasmas de testículos, mama, esôfago, rim, tireoide, lábio, cabeça e pescoço e osso.

PALAVRAS-CHAVE Câncer. Agroquímicos. Doenças profissionais. Revisão. 


\section{Introduction}

Pesticides are chemical substances or mixture of substances also used in the public health domain so to combat disease vectors, such as mosquitoes, as in agriculture to combat pests that harm crops ${ }^{1}$. Although they form the base of modern agriculture, pesticides are associated with chemical contamination, which is a complex public and environmental health problem, especially in the rural area ${ }^{\mathbf{2}, \mathbf{3}}$.

Most sprayed pesticides reach non-target species and end up polluting air, water and soil, soon contaminating the pesticide applicators, their direct family, as well as other people living in agricultural areas, who consume foods with high concentrations of these substances ${ }^{4-6}$.

Studies have related exposure to pesticides to cancer ${ }^{7}$, a chronic disease that is one of the main causes of morbidity and mortality worldwide, with over 14 million new cases in $2012^{8}$. In 2015 , 8.8 million people worldwide died due to malignant neoplasms, the equivalent to one in six of all global deaths'.

Many review papers, available on Medline/ PubMed database under the search described below, investigated the relation between pesticide and cancer. However, they either reviewed only (a) one type of cancer, (b) one type of pesticide or chemical group, (c) one study design or research group, (d) one age range, or (e) a sole population. Therefore, the aim of this study was to conduct an integrative literature review of published studies on pesticide exposure and cancer with a focus on farmers, rural population, pesticide applicators and rural workers, considering all cancer types, agricultural pesticides, and age ranges.

\section{Methods}

Studies were retrieved from the Medline/ PubMed database (https://www.ncbi. nlm.nih.gov/pubmed/advanced) using the following key words in English and Portuguese: cancer OR carcinogenic OR tumor OR cancer OR carcinogenic OR neoplasia AND pesticide OR herbicide OR insecticide OR fungicide OR organophosphate OR agrochemical OR pesticide OR herbicide OR insecticide OR fungicidal OR organofosforados OR agrotoxicos OR agroquimico AND farmers OR husbandman OR agriculturists OR agriculturalists OR agricultural OR cultivator OR applicator OR agriculture OR "rural people" OR "rural population" OR "rural areas" OR "non-urban" OR rural OR "trabalhador rural” OR agricola OR applicator OR "populacao rural" OR "areas rurais" AND cohort OR "case-control" OR "case control" OR transversal OR "medical record" OR "ecological design" OR "ecologic design" OR "ecologic study" OR coorte OR "caso-controle" OR "caso controle" OR prontuario OR "delineamento ecologico".

Original articles published between August 2007 and August 2017 and examining the relationship between pesticides and cancer were included in this review. Studies were excluded whenever they (a) were not related to farmers, rural population, agricultural pesticide applicators, rural workers, or to residents of areas with intensive use of agricultural pesticides; (b) did not analyze cancer or pesticide; (c) were reviews; (d) analyzed pesticide intake through food; (e) focused on analyses of biomarkers or dust; (f) concerned genetic studies; (g) were not written in English or Portuguese; or (h) had a focus on methodology or protocol.

A primary screening of the titles and abstracts was carried out in order to remove records that fit the excluding criteria. A second and deeper screening analyzed the full text. After the evaluation, 74 studies were chosen to compose the accepted sample (figure 1). The discussion was organized according to overall cancers and specific cancer types so as to better investigate the relationship with pesticide exposure. 
Figure 1. Flowchart of the studies included in this integrative review

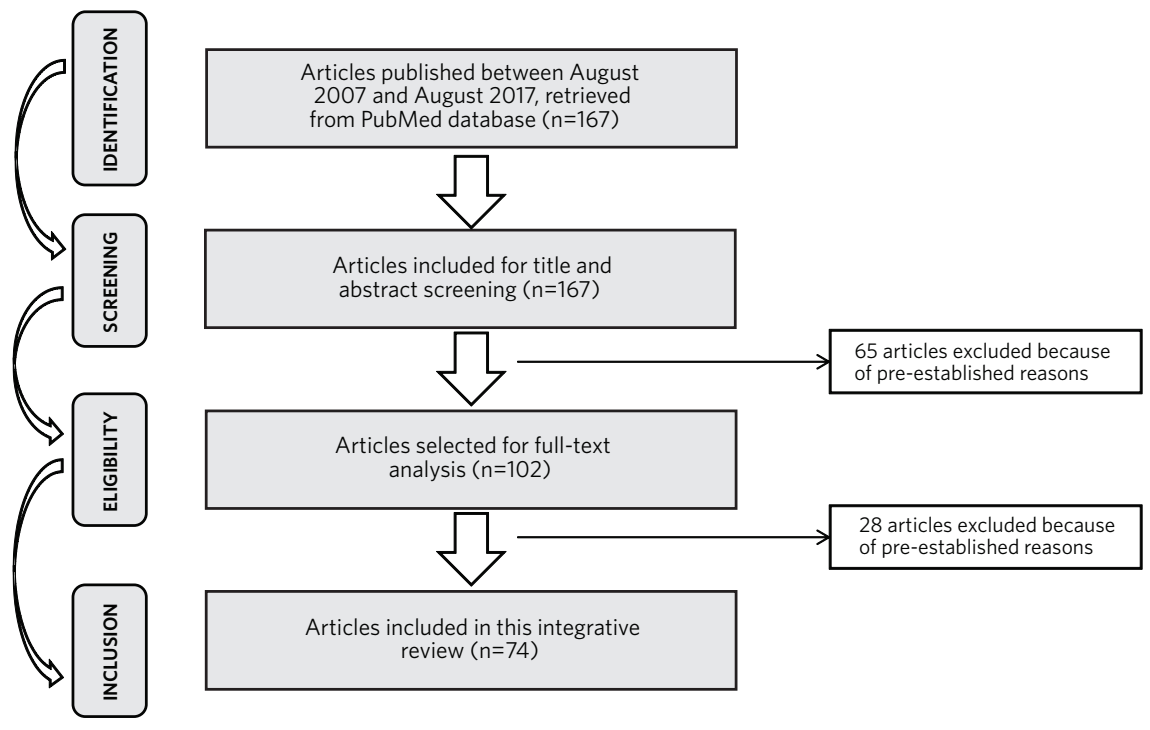

Source: Own elaboration

\section{Results}

The search on Medline/PubMed database resulted in 167 papers, of which 74 were selected for this study (chart 1). Findings were summarized according to individual cancer types. Several specific pesticides were related to increased risk of cancer and are listed in chart 2. The vast majority of the papers reviewed concerned to either case-control (39) or cohort (32) studies. Only one study applied a cross-sectional design and two others, an ecological outline. Overall, 64 papers observed a relationship between pesticides and cancer while 10 could not find any significant positive association. Chart 3 shows the registration status of pesticides in the European Union, the United States, and Brazil.

Chart 1. Summary of studies selected for this review

\begin{tabular}{|c|c|c|c|c|}
\hline Cancer type & Study design & Sample size & Place/Country of study & References \\
\hline Bladder and colon & Cohort & 20,646 & IA and NC, USA & Koutros et al. (2009)72 \\
\hline Bladder & Cohort & 54,344 & IA and NC, USA & Koutros et al. $(2016)^{73}$ \\
\hline Bladdera & Cohort & 148,051 & France & Boulanger et al. (2017) $\mathbf{7 4}$ \\
\hline Brain & Case-control & $\begin{array}{l}2,040 \text { cases }+4,140 \text { con- } \\
\text { trols }\end{array}$ & RJ, Brazil & $\begin{array}{l}\text { Miranda-Filho et al. } \\
(2012)^{65}\end{array}$ \\
\hline Brain & Cohort & 7,734 & RJ, Brazil & $\begin{array}{l}\text { Miranda Filho et al. } \\
\text { (2014)66 }\end{array}$ \\
\hline Breasta & Case-control & 207 cases +621 controls & Canada & $\begin{array}{l}\text { Ashley-Martin et al. } \\
(2012)^{\mathbf{7 0}}\end{array}$ \\
\hline Cervical & Case-control & 33 cases +132 controls & Wuhan, China & Zhang et al. (2013)69 \\
\hline Cholangio carcinomaa & Case-control & 210 cases +840 controls & Thailand & Jeephet et al. (2016)63 \\
\hline
\end{tabular}




\begin{tabular}{|c|c|c|c|c|}
\hline CNSb & Cohort & 181,842 & France & Piel et al. (2017)64 \\
\hline Colon & Cohort & 25,712 & IA and NC, USA & Kang et al. (2008)57 \\
\hline Colon and breast & Cohort & $\begin{array}{l}39,628 \text { men + 28,319 } \\
\text { women }\end{array}$ & IA and NC, USA & Andreotti et al. (2010)58 \\
\hline Colorretal & Case-control & 421 cases +439 controls & Egypt & Lo et al. $(2010)^{56}$ \\
\hline Cutaneous melanoma & Case-control & 150 cases $+24,554$ controls & IA and NC, USA & Dennis et al. (2010)77 \\
\hline Esophagus & Case-control & 5,782 cases $+5,782$ controls & RS, PR, SC, Brazil & Meyer et al. (2011)59 \\
\hline Glioma & Case-control & 798 cases $+1,175$ controls & IA, MI, MN, and WI, USA & Ruder et al. (2009)67 \\
\hline Gliomaa & Case-control & 798 cases $+1,175$ controls & IA, MI, MN, and WI, USA & Yiin et al. (2012)68 \\
\hline $\mathrm{HCC}$ & Case-control & $\begin{array}{l}3,034 \text { cases }+14,991 \text { con- } \\
\text { trols }\end{array}$ & CA, USA & Vopham et al. (2015)61 \\
\hline Head and neck & Case-control & 7 cases +5 controls & Oklahoma, USA & Govett et al. (2011)81 \\
\hline$H L^{d}$ & Case-control & 316 cases $+1,506$ controls & 6 provinces, Canada & Pahwa et al. (2009)33 \\
\hline$H L^{d}$ & Case-control & 316 cases $+1,506$ controls & 6 provinces, Canada & $\begin{array}{l}\text { Karunanayake et al. } \\
(2012)^{32}\end{array}$ \\
\hline Leukemia & Case-control & 252 cases +423 controls & 13 states, Brazil & Ferreira et al. (2013) 26 \\
\hline Leukemia & Cohort & $6,479,406$ & South Korea & Cha et al. (2014)30 \\
\hline Leukemia & Case-control & 132 cases +132 controls & Rohtak, India & Kumar et al. (2014)27 \\
\hline Leukemia & Ecologic & Not applicable & 6 states, USA & Booth et al. $(2015)^{\mathbf{2 8}}$ \\
\hline Leukemiaa & Case-control & 111 casos +444 controls & 2 provinces, Italy & Malagoli et al. (2016)29 \\
\hline Leukemia (ALLe) & Case-control & 213 cases +268 controls & CA, USA & Rull et al. (2009)25 \\
\hline Leukemia $\left(A M L^{f}\right)$ & Case-control & 722 cases $+1,444$ controls & Shanghai, China & Wong et al. (2009)31 \\
\hline Liver & Case-control & 281 cases +20 controls & Tanta, Egypt & Azm et al. (2014)60 \\
\hline $\begin{array}{l}\text { Liver and follicular cell } \\
\text { lymphoma }\end{array}$ & Cohort & 49,616 & IA and NC, USA & Silver et al. (2015)62 \\
\hline Lung & Cohort & 22,830 & IA and NC, USA & Jones et al. (2015)21 \\
\hline Lung & Case-control & $\begin{array}{l}546 \text { cases }+49,266 \text { con- } \\
\text { trols }\end{array}$ & IA and NC, USA & Bonner et al. (2017)76 \\
\hline LHCg & Cohort & 23,072 & IA and NC, USA & Delancey et al. (2009)22 \\
\hline LHCg & Case-control & 354 cases +455 controls & Tessalia, Greece & Kokouva et al. (2011)23 \\
\hline LHCg & Cohort & 37,099 & IA, USA & Jones et al. (2014) \\
\hline LHCg & Cohort & 76,493 & USA & Schinasi et al. (2015) $\mathbf{2 4}$ \\
\hline Melanoma & Cohort & 21,416 & IA and NC, USA & Mahajan et al. (2007) ${ }^{\mathbf{7 8}}$ \\
\hline MDSh & Case-control & 126 cases +102 controls & Greece & Avgerinou et al. (2017)83 \\
\hline MMi & Cohort & $2,992,166$ & Sweden & Lope et al. (2008)47 \\
\hline MMi & Cohort & 49,093 & IA and NC, USA & Rusiecki et al. (2009)46 \\
\hline MMi & Case-control & 342 cases $+1,506$ controls & 6 provinces, Canada & Pahwa et al. (2012) 44 \\
\hline MMi & Case-control & 342 cases $+1,357$ controls & 6 provinces, Canada & Kachuri et al. (2013) 43 \\
\hline MMi & Case-control & 547 cases $+2,700$ controls & USA, Canada & Presutti et al. (2016)45 \\
\hline$N H L^{j}$ & Case-control & 858 cases $+1,821$ controls & Germany & $\begin{array}{l}\text { Richardson et al. } \\
(2008)^{35}\end{array}$ \\
\hline NHLi & Cohort & 56,222 & IA and NC, USA & Park et al. (2009)42 \\
\hline
\end{tabular}




\begin{tabular}{|c|c|c|c|c|}
\hline NHLj & Case-control & 649 cases $+1,298$ controls & Shanghai, China & Wong et al. (2010)31 \\
\hline$N H L^{j}$ & Case-control & 513 cases $+1,506$ controls & 6 provinces, Canada & Hohenadel et al. (2011)40 \\
\hline $\mathrm{NHLi}$ & Case-control & 75 cases +321 controls & Saskatchewan, Canada & $\begin{array}{l}\text { Karunanayake et al. } \\
(2013)^{\mathbf{3 9}}\end{array}$ \\
\hline$N H L^{j}$ & Cohort & 54,306 & IA and NC, USA & Alavanja et al. (2014)41 \\
\hline $\mathrm{NHLj}$ & Case-control & 1,317 cases $+2,634$ controls & Brazil & Boccolini et al. (2016)36 \\
\hline Pancreatic & Case-control & 93 cases $+82,503$ controls & IA and NC, USA & Andreotti et al. (2009)54 \\
\hline Prostate & Cohort & 47,822 & IA and NC, USA & $\begin{array}{l}\text { Christensen et al. } \\
\text { (2010)52 }\end{array}$ \\
\hline Prostate & Case-control & 1,153 cases $+3,999$ controls & Canada & Band et al. (2011)50 \\
\hline Prostate & Case-control & 173 cases +162 controls & CA, USA & Cockburn et al. (2011)49 \\
\hline Prostate & $\begin{array}{l}\text { Cross-sec- } \\
\text { tional }\end{array}$ & 2,938 & Saskatchewan, Canada & Sharma et al. (2016)51 \\
\hline Several typesa & Cohort & 19,717 & IA and NC, USA & Bonner et al. (2007) ${ }^{\mathbf{1 4}}$ \\
\hline Several types & Ecologic & $25,110,289$ & USA & Carozza et al. (2008)17 \\
\hline Several types ${ }^{a}$ & Cohort & 49,762 & IA and NC, USA & Koutros et al. (2008)15 \\
\hline Several typesa & Cohort & 47,625 & IA and NC, USA & $\begin{array}{l}\text { Mozzachio et al. } \\
(2008)^{\mathbf{1 2}}\end{array}$ \\
\hline Several typesa & Cohort & 48,986 & IA and NC, USA & $\begin{array}{l}\text { Greenburg et al. } \\
(2008)^{13}\end{array}$ \\
\hline Several types & Cohort & 48,378 & IA and NC, USA & $\begin{array}{l}\text { Van Bemmel et al. } \\
(2008)^{9}\end{array}$ \\
\hline Several types & Case-control & 1,778 cases $+1,802$ controls & TX, USA & Carozza et al. (2009)18 \\
\hline Several types & Cohort & 19,655 & IA and NC, USA & Lynch et al. (2009)10 \\
\hline Several types & Cohort & 44,624 & IA and NC, USA & Bonner et al. (2010)11 \\
\hline Several types & Cohort & 62,960 & Great Britain & Frost el al. (2011)48 \\
\hline Several types & Case-control & $\begin{array}{l}34,205 \text { cases }+1,832,969 \\
\text { controls }\end{array}$ & Andalusia, Spain & Parrón et al. (2014) $\mathbf{3 4}$ \\
\hline Several types & Cohort & 30,003 & $I A$ and $N C$, USA & Lerro et al. (2015)71 \\
\hline Several types & Case-control & 887 cases $+11,491$ controls & Italy & Salerno et al. (2016)19 \\
\hline Several types & Case-control & $\begin{array}{l}3,350 \text { cases }+20,365 \\
\text { controls }\end{array}$ & Spain & $\begin{array}{l}\text { Gómez-Barroso et al. } \\
(2016)^{\mathbf{1 6}}\end{array}$ \\
\hline Several types & Cohort & 70,570 & Canada & Kachuri et al. (2017)37 \\
\hline Several types & Cohort & 181,842 & France & $\begin{array}{l}\text { Lemarchand et al. } \\
(2017)^{\mathbf{2 0}}\end{array}$ \\
\hline Stomach & Cohort & 53,588 & IA and NC, USA & Barry et al. $(2012)^{55}$ \\
\hline STSk & Case-control & 357 cases $+1,506$ controls & 6 provinces, Canada & Pahwa et al. (2011) ${ }^{80}$ \\
\hline Thyroid & Cohort & 36,357 & IA and NC, USA & Freeman et al., (2011)82 \\
\hline Uveal melanomaa & Case-control & 293 cases $+3,198$ controls & 9 European countries ${ }^{1}$ & Behrens et al. (2012)79 \\
\hline
\end{tabular}

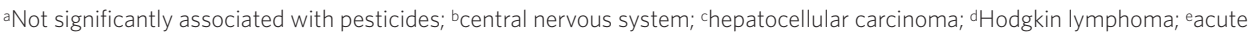
lymphoblastic leukemia; facute myeloid leukemia; glymphohematopoietic cancer; hmyelodysplastic syndromes; imultiple myeloma; inonHodgkin lymphoma; ksoft tissue sarcoma; 'Denmark, Latvia, France, Germany, Italy, Sweden, Spain, Portugal, and UK. 
Chart 2. Pesticides positively associated with cancer among studies that presented Odd Ratios, Relative Risks, or Hazard Ratios

\begin{tabular}{|c|c|c|c|c|c|c|c|}
\hline $\begin{array}{l}\text { Cancer type } \\
\text { associated }\end{array}$ & Pesticide & $\begin{array}{l}\text { Pesticide } \\
\text { chemical groupe }\end{array}$ & $\begin{array}{l}\text { Pesticide } \\
\text { according } \\
\text { to the pest } \\
\text { it controls }\end{array}$ & $\begin{array}{l}\text { RR, OR, or HR with } \\
95 \% \text { confidence } \\
\text { intervalg }\end{array}$ & $\begin{array}{l}p \text {-value } \\
\text { for } \\
\text { linear } \\
\text { trend }\end{array}$ & Comparison groups ${ }^{h}$ & References \\
\hline All types & EPTC & Thiocarbamate & Herbicide & $\mathrm{RR}=1.28(1.09-1.50)$ & $<0.01$ & $\begin{array}{l}\text { Highly exposed ( } \geq 50 \text { LD) vs } \\
\text { non-exposed }\end{array}$ & $\begin{array}{l}\text { Van Bemmel et al. } \\
\text { (2008) }\end{array}$ \\
\hline All types & Butylate & Thiocarbamate & Herbicide & $R R=1.70(1.20-2.40)$ & 0.01 & $\begin{array}{l}\text { Highly exposed ( } \geq 57 \text { LD) vs low } \\
\text { exposed (1-9 LD) }\end{array}$ & Lynch et al. (2009) \\
\hline All types & Terbufos & $\begin{array}{l}\text { Organophos- } \\
\text { phate }\end{array}$ & Insecticide & $H R=1.21(1.06-1.37)$ & $>0.05$ & $\begin{array}{l}\text { Highly exposed (>352 IWLD) vs } \\
\text { non-exposed }\end{array}$ & Bonner et al. (2010) \\
\hline Bladder & Imazethapyr & Imidazolinone & Herbicide & $R R=2.37(1.20-4.68)$ & 0.01 & $\begin{array}{l}\text { T3, upper half ( } \geq 311.9 \text { IWLD) vs } \\
\text { non-exposed }\end{array}$ & Koutros et al. (2009) \\
\hline Bladder & Imazaquin & Imidazolinone & Herbicide & $R R=1.54(1.05-2.26)$ & $<0.05$ & Ever vs never use & Koutros et al. (2016) \\
\hline Bladder & Bentazon & Thiadiazinol & Herbicide & $\mathrm{RR}=1.55(1.10-2.19)$ & $<0.05$ & Ever vs never use & Koutros et al. (2016) \\
\hline Bladder & Bromoxynil & Nitrile & Herbicide & $\mathrm{RR}=1.51(1.04-2.20)$ & $<0.05$ & Ever vs never use & Koutros et al. (2016) \\
\hline Bladder & Chloramben & Benzoic acid & Herbicide & $\mathrm{RR}=1.56(1.10-2.22)$ & $<0.05$ & Ever vs never use & Koutros et al. (2016) \\
\hline Bladder & $\begin{array}{l}\text { Diclofop- } \\
\text { methyl }\end{array}$ & $\begin{array}{l}\text { Chlorinated } \\
\text { phenol }\end{array}$ & Herbicide & $\mathrm{RR}=1.85(1.01-3.42)$ & $<0.05$ & Ever vs never use & Koutros et al. (2016) \\
\hline Bladder & DDT & Organochlorine & Insecticide & $\mathrm{RR}=1.40(1.10-1.80)$ & $<0.05$ & Ever vs never use & Koutros et al. (2016) \\
\hline Bladder & Imazethapyr & Imidazolinone & Herbicide & $R R=3.03(1.46-6.29)$ & 0.004 & $\begin{array}{l}\text { Q4 vs non-exposed, among never } \\
\text { smokers }\end{array}$ & Koutros et al. (2016) \\
\hline Bladder & $2,4,5-\mathrm{T}$ & $\begin{array}{l}\text { Chlorinated } \\
\text { phenol }\end{array}$ & Herbicide & $R R=2.64(1.23-5.68)$ & 0.02 & $\begin{array}{l}\text { T3 vs non-exposed, among never } \\
\text { smokers }\end{array}$ & Koutros et al. (2016) \\
\hline Bladder & $2,4-D$ & $\begin{array}{l}\text { Chlorinated } \\
\text { phenol }\end{array}$ & Herbicide & $R R=1.88(0.94-3.77)$ & 0.02 & $\begin{array}{l}\text { Q4 vs non-exposed, among never } \\
\text { smokers }\end{array}$ & Koutros et al. (2016) \\
\hline Bladder & & Glyphosate & Herbicide & $R R=1.93(0.95-3.91)$ & 0.03 & $\begin{array}{l}\text { Q4 vs non-exposed, among never } \\
\text { smokers }\end{array}$ & Koutros et al. (2016) \\
\hline Breast & & $\begin{array}{l}\text { Organophos- } \\
\text { phate }\end{array}$ & Insecticide & $R R=1.20(1.01-1.43)$ & & Ever vs never use & Lerro et al. (2015) \\
\hline Colon & Trifluralin & Dinitroaniline & Herbicide & $R R=1.76(1.05-2.95)$ & 0.036 & T3 (upper half) vs non-exposed & Kang et al. (2008) \\
\hline Colon & EPTC & Thiocarbamate & Herbicide & $R R=2.09(1.26-3.47)$ & $<0.01$ & $\begin{array}{l}\text { Highly exposed ( } \geq 50 \text { LD) vs } \\
\text { non-exposed }\end{array}$ & $\begin{array}{l}\text { Van Bemmel et al. } \\
\text { (2008) }\end{array}$ \\
\hline Colon & Imazethapyr & Imidazolinone & Herbicide & $\mathrm{RR}=1.78(1.08-2.93)$ & 0.02 & T3 (upper half) vs non-exposed & Koutros et al. (2009) \\
\hline Colon & Carbofuran & Carbamate & Insecticide & $H R=1.10(1.04-1.17)$ & & Ever vs never use among males & Andreotti et al. (2010) \\
\hline Colon & Metolachlor & Chloroacetanilide & Herbicide & $H R=1.09(1.04-1.15)$ & & Ever vs never use among males & Andreotti et al. (2010) \\
\hline Colon & Alachlor & Chloroacetanilide & Herbicide & $H R=1.08(1.03-1.13)$ & & Ever vs never use among males & Andreotti et al. (2010) \\
\hline $\begin{array}{l}\text { Cutaneous } \\
\text { Melanoma }\end{array}$ & Carbaryl & Carbamate & Insecticide & $\mathrm{OR}=1.7(1.1-2.5)$ & 0.013 & $\begin{array}{l}\text { Highly exposed ( } \geq 56 \text { LD) vs } \\
\text { non-exposed }\end{array}$ & Dennis et al. (2010) \\
\hline $\begin{array}{l}\text { Cutaneous } \\
\text { Melanoma }\end{array}$ & Parathion & $\begin{array}{l}\text { Organophos- } \\
\text { phate }\end{array}$ & Insecticide & $\mathrm{OR}=2.4(1.3-4.4)$ & 0.003 & $\begin{array}{l}\text { Highly exposed ( } \geq 56 \text { LD) vs } \\
\text { non-exposed }\end{array}$ & Dennis et al. (2010) \\
\hline $\begin{array}{l}\text { Cutaneous } \\
\text { Melanoma }\end{array}$ & $\begin{array}{l}\text { Maneb/ man- } \\
\text { cozeb }\end{array}$ & Dithiocarbamate & Fungicide & $\mathrm{OR}=2.4(1.2-4.9)$ & 0.006 & $\begin{array}{l}\text { Highly exposed ( } \geq 63 \text { LD) vs } \\
\text { non-exposed }\end{array}$ & Dennis et al. (2010) \\
\hline $\begin{array}{l}\text { Follicular cell } \\
\text { lymphoma }\end{array}$ & Metolachlor & Chloroacetanilide & Herbicide & $R R=2.89(1.13-7.38)$ & 0.03 & Q4 (>108.5 LD) vs non-exposed & Silver et al. (2015) \\
\hline $\begin{array}{l}\text { Hodgkin } \\
\text { lymphoma }\end{array}$ & Chlorpyrifos & $\begin{array}{l}\text { Organophos- } \\
\text { phate }\end{array}$ & Insecticide & $\mathrm{OR}=5.26(1.56-17.79)$ & & Exposed vs non-exposed & $\begin{array}{l}\text { Karunanayake et al. } \\
\text { (2012) }\end{array}$ \\
\hline $\begin{array}{l}\text { Hodgkin } \\
\text { lymphoma }\end{array}$ & Dichlorprop & Chlorophenoxy & Herbicide & $\mathrm{OR}=6.35(1.56-25.92)$ & & Exposed vs non-exposed & Pahwa et al. (2009) \\
\hline $\begin{array}{l}\text { Hepatocel- } \\
\text { lular carci- } \\
\text { noma }\end{array}$ & & Organochlorine & Insecticide & $\mathrm{OR}=1.87(1.17-2.99)$ & & Q4 (>14.53 kg km-2) vs others & Vopham et al. (2015) \\
\hline
\end{tabular}




\section{Chart 2. (cont.)}

\begin{tabular}{|c|c|c|c|c|c|c|c|}
\hline Leukemia & EPTC & Thiocarbamate & Herbicide & $R R=2.36(1.16-4.84)$ & 0.02 & $\begin{array}{l}\text { Highly exposed ( } \geq 50 \text { LD) vs } \\
\text { non-exposed }\end{array}$ & $\begin{array}{l}\text { van Bemmel et al. } \\
\text { (2008) }\end{array}$ \\
\hline Leukemia & Terbufos & $\begin{array}{l}\text { Organophos- } \\
\text { phate }\end{array}$ & Insecticide & $H R=2.38(1.35-4.21)$ & $>0.05$ & $\begin{array}{l}\text { Moderately exposed } \\
(107<\mid \text { WLD }>352) \text { vs non- } \\
\text { exposed }\end{array}$ & Bonner et al. (2010) \\
\hline $\begin{array}{l}\text { Leukemia } \\
\left(A L L^{a}\right)\end{array}$ & & $\begin{array}{l}\text { Organophos- } \\
\text { phate }\end{array}$ & Insecticide & $\mathrm{OR}=1.6(1.0-2.7)$ & & $\begin{array}{l}\text { Moderately exposed ( } 1-79 \mathrm{lb} / \\
\text { mi2) vs low exposure }(<1 \mathrm{lb} / \mathrm{mi} 2)\end{array}$ & Rull et al. (2009) \\
\hline $\begin{array}{l}\text { Leukemia } \\
\left(A L L^{a}\right)\end{array}$ & & $\begin{array}{l}\text { Chlorinated } \\
\text { phenol }\end{array}$ & & $\mathrm{OR}=2.0(1.0-3.8)$ & & $\begin{array}{l}\text { Moderately exposed (1-7 lb/mi2) } \\
\text { vs low exposure (<1 lb/mi2) }\end{array}$ & Rull et al. (2009) \\
\hline $\begin{array}{l}\text { Leukemia } \\
\left(A L L^{a}\right)\end{array}$ & & Triazine & Herbicide & $\mathrm{OR}=1.9(1.0-3.7)$ & & $\begin{array}{l}\text { Moderately exposed (1-27lb/ } \\
\text { mi2) vs low exposure }(<1 \mathrm{lb} / \mathrm{mi} 2)\end{array}$ & Rull et al. (2009) \\
\hline $\begin{array}{l}\text { Leukemia } \\
\left(A L L^{a}\right)\end{array}$ & & & Fumigant & $\mathrm{OR}=1.7(1.0-3.1)$ & & $\begin{array}{l}\text { Moderately exposed (1-549 lb/ } \\
\text { mi2) vs low exposure (<1 lb/mi2) }\end{array}$ & Rull et al. (2009) \\
\hline $\begin{array}{l}\text { Leukemia } \\
\left(A L L^{a}\right)\end{array}$ & Permethrin & Pyrethroid & Insecticide & $\mathrm{OR}=2.47(1.17-5.25)$ & & Children up to 11 months & Ferreira et al. (2013) \\
\hline $\begin{array}{l}\text { Leukemia } \\
\left(A L L^{a}\right)\end{array}$ & Imiprothrin & Pyrethroid & Insecticide & $\mathrm{OR}=2.61(1.06-6.93)$ & & Children up to 11 months & Ferreira et al. (2013) \\
\hline $\begin{array}{l}\text { Leukemia } \\
\left(A L L^{a}\right)\end{array}$ & Esbiothrin & Pyrethroid & Insecticide & $\mathrm{OR}=3.03(1.13-8.09)$ & & Children up to 11 months & Ferreira et al. (2013) \\
\hline $\begin{array}{l}\text { Leukemia } \\
\left(A M L^{b}\right)\end{array}$ & Prallethrin & Pyrethroid & Insecticide & $\mathrm{OR}=8.06(1.17-55.65)$ & & Children up to 11 months & Ferreira et al. (2013) \\
\hline $\begin{array}{l}\text { Leukemia } \\
\left(\mathrm{AML}{ }^{b}\right)\end{array}$ & Permethrin & Pyrethroid & Insecticide & $\mathrm{OR}=7.28(2.60-20.38)$ & & Children up to 11 months & Ferreira et al. (2013) \\
\hline $\begin{array}{l}\text { Leukemia } \\
\left(\mathrm{AML} \mathrm{L}^{\mathrm{b}}\right)\end{array}$ & Tetramethrin & Pyrethroid & Insecticide & OR=6.19 (2.07-18.56) & & Children up to 11 months & Ferreira et al. (2013) \\
\hline $\begin{array}{l}\text { Leukemia } \\
\left(A M L^{b}\right)\end{array}$ & d-Allethrin & Pyrethroid & Insecticide & OR=6.19 (2.07-18.56) & & Children up to 11 months & Ferreira et al. (2013) \\
\hline $\begin{array}{l}\text { Leukemia } \\
\left(\mathrm{AML} \mathrm{L}^{\mathrm{b}}\right)\end{array}$ & Esbiothrin & Pyrethroid & Insecticide & $\mathrm{OR}=3.71(1.18-11.62)$ & & $\begin{array}{l}\text { Children between } 12 \text { and } 23 \\
\text { months }\end{array}$ & Ferreira et al. (2013) \\
\hline $\begin{array}{l}\text { Leukemia } \\
\left.(\mathrm{AML})^{\mathrm{b}}\right)\end{array}$ & d-phenothrin & Pyrethroid & Insecticide & $\mathrm{OR}=8.43(1.59-44.75)$ & & $\begin{array}{l}\text { Children between } 12 \text { and } 23 \\
\text { months }\end{array}$ & Ferreira et al. (2013) \\
\hline $\mathrm{LHCc}$ & Butylate & Thiocarbamate & Herbicide & $R R=1.84(1.14-2.97)$ & 0.01 & $\begin{array}{l}\text { Highly exposed ( } \geq 26 \text { LD) vs non- } \\
\text { exposed }\end{array}$ & Lynch et al. (2009) \\
\hline $\mathrm{LHCc}^{\mathrm{C}}$ & Metribuzin & Triazole & Herbicide & $R R=2.07(0.99-4.29)$ & 0.02 & $\begin{array}{l}\text { Highly exposed ( } \geq 174.4 \text { IWLD) vs } \\
\text { low exposed }\end{array}$ & $\begin{array}{l}\text { Delancey et al. } \\
\text { (2009) }\end{array}$ \\
\hline LHCc & Terbufos & $\begin{array}{l}\text { Organophos- } \\
\text { phate }\end{array}$ & Insecticide & $H R=1.85(1.31-2.62)$ & $>0.05$ & $\begin{array}{l}\text { Moderately exposed } \\
(107<\mid \text { WLD }>352) \text { vs non- } \\
\text { exposed }\end{array}$ & Bonner et al. (2010) \\
\hline Liver & Metolachlor & Chloroacetanilide & Herbicide & $R R=3.99(1.43-11.1)$ & $<0.01$ & Q4 (>108.5 LD) vs non-exposed & Silver et al. (2015) \\
\hline Lungs & Diazinon & $\begin{array}{l}\text { Organophos- } \\
\text { phate }\end{array}$ & Insecticide & $\mathrm{RR}=1.60(1.11-2.31)$ & 0.02 & $\begin{array}{l}\text { Highly exposed (>38.8 LD) vs } \\
\text { non-exposed }\end{array}$ & Jones et al. (2015) \\
\hline Lungs & $\begin{array}{l}\text { Chlorimuron } \\
\text { ethyl }\end{array}$ & Sulfenylurea & Herbicide & $H R=1.74(1.02-2.96)$ & 0.18 & $\begin{array}{l}\text { Fourth quartile vs non-exposed, } \\
\text { based on LD }\end{array}$ & Bonner et al. (2017) \\
\hline Melanoma & Carbaryl & Carbamate & Insecticide & $R R=3.55(1.27-9.96)$ & 0.07 & $\begin{array}{l}\text { Moderately exposed (57-175 LD) } \\
\text { vs non-exposed }\end{array}$ & Mahajan et al. (2007) \\
\hline Melanoma & Carbaryl & Carbamate & Insecticide & $R R=4.11(1.33-12.75)$ & 0.07 & $\begin{array}{l}\text { Highly exposed (>175 LD) vs } \\
\text { non-exposed }\end{array}$ & Mahajan et al. (2007) \\
\hline $\begin{array}{l}\text { Myelo- } \\
\text { dysplastic } \\
\text { syndromes }\end{array}$ & Paraquat & Organic & Herbicide & $\mathrm{OR}=4.90(1.05-22.75)$ & & Exposed vs non-exposed & $\begin{array}{l}\text { Avgerinou et al. } \\
\text { (2017) }\end{array}$ \\
\hline $\begin{array}{l}\text { Multiple } \\
\text { Myeloma }\end{array}$ & Captan & Phentolamine & Fungicide & $\mathrm{OR}=2.35(1.03-5.35)$ & & Exposed vs non-exposed & Pahwa et al. (2012) \\
\hline $\begin{array}{l}\text { Multiple } \\
\text { Myeloma }\end{array}$ & & Carbamate & Insecticide & OR=1.90 (1.11-3.27) & & Exposed vs non-exposed & Pahwa et al. (2012) \\
\hline
\end{tabular}




\begin{tabular}{|c|c|c|c|c|c|c|c|}
\hline \multicolumn{8}{|c|}{ Chart 2. (cont.) } \\
\hline $\begin{array}{l}\text { Multiple } \\
\text { Myeloma }\end{array}$ & Mecoprop & Phenoxy & Herbicide & $\mathrm{OR}=1.89(1.15-3.12)$ & & Exposed vs non-exposed & Pahwa et al. (2012) \\
\hline $\begin{array}{l}\text { Multiple } \\
\text { Myeloma }\end{array}$ & Mecoprop & Phenoxy & Herbicide & $\mathrm{OR}=1.94(1.19-3.19)$ & & Exposed vs non-exposed & Kachuri et al. (2013) \\
\hline $\begin{array}{l}\text { Multiple } \\
\text { Myeloma }\end{array}$ & Carbaryl & Carbamate & Insecticide & $\mathrm{OR}=2.71(1.47-5.00)$ & & Exposed vs non-exposed & Kachuri et al. (2013) \\
\hline $\begin{array}{l}\text { Multiple } \\
\text { Myeloma }\end{array}$ & Lindane & Organochlorine & Insecticide & $\mathrm{OR}=2.37(1.08-5.16)$ & & Exposed vs non-exposed & Kachuri et al. (2013) \\
\hline $\begin{array}{l}\text { Multiple } \\
\text { Myeloma }\end{array}$ & Captan & Phentolamine & Fungicide & $\mathrm{OR}=2.96(1.40-6.24)$ & & Exposed vs non-exposed & Kachuri et al. (2013) \\
\hline $\begin{array}{l}\text { Multiple } \\
\text { Myeloma }\end{array}$ & Carbaryl & Carbamate & Insecticide & $\mathrm{OR}=2.02(1.28-3.21)$ & & Ever vs never use & Presutti et al. (2016) \\
\hline $\begin{array}{l}\text { Multiple } \\
\text { Myeloma }\end{array}$ & Captan & Phentolamine & Fungicide & $\mathrm{OR}=1.98(1.04-3.77)$ & & Ever vs never use & Presutti et al. (2016) \\
\hline $\begin{array}{l}\text { Multiple } \\
\text { Myeloma }\end{array}$ & DDT & Organochlorine & Insecticide & $\mathrm{OR}=1.44(1.05-1.97)$ & & Ever vs never use & Presutti et al. (2016) \\
\hline $\begin{array}{l}\text { Multiple } \\
\text { Myeloma }\end{array}$ & Permethrin & Pyrethroid & Insecticide & $R R=3.1(1.5-6.2)$ & 0.002 & $\begin{array}{l}\text { Highly exposed (>50.75 LD) vs } \\
\text { non-exposed }\end{array}$ & Alavanja et al. (2014) \\
\hline $\begin{array}{l}\text { Multiple } \\
\text { Myeloma }\end{array}$ & Permethrin & Pyrethroid & Insecticide & $\mathrm{RR}=5.72(2.76-11.87)$ & $<0.01$ & $\begin{array}{l}\text { Highly exposed (> } 50.75 \text { LD) vs } \\
\text { non-exposed }\end{array}$ & Rusiecki et al. (2009) \\
\hline $\mathrm{NHL}$ & Paraquat & Organic & Herbicide & $R R=1.51(1.01-2.26)$ & & Ever vs never used & Park et al. (2009) \\
\hline$N H L^{d}$ & Butylate & Thiocarbamate & Herbicide & $R R=2.94(1.49-5.76)$ & 0.002 & $\begin{array}{l}\text { Highly exposed ( } \geq 26 \text { LD) vs } \\
\text { non-exposed }\end{array}$ & Lynch et al. (2009) \\
\hline$N H L^{d}$ & Terbufos & $\begin{array}{l}\text { Organophos- } \\
\text { phate }\end{array}$ & Insecticide & $H R=1.94(1.16-3.22)$ & $>0.05$ & $\begin{array}{l}\text { Moderately exposed } \\
(107<\mid \text { WLD>352) vs non- } \\
\text { exposed }\end{array}$ & Bonner et al. (2010) \\
\hline$N H L^{d}$ & All pesticides & & & $\mathrm{OR}=1.63(1.20-2.21)$ & 0.01 & $\begin{array}{l}\text { Highly exposed ( } \geq 5 \text { pesticides) vs } \\
\text { non-exposed }\end{array}$ & $\begin{array}{l}\text { Hohenadel et al. } \\
\text { (2011) }\end{array}$ \\
\hline$N H L^{d}$ & & & Herbicide & $\mathrm{OR}=1.62(1.18-2.22)$ & 0.02 & $\begin{array}{l}\text { Moderately exposed ( } 2-4 \text { pesti- } \\
\text { cides) vs non-exposed }\end{array}$ & $\begin{array}{l}\text { Hohenadel et al. } \\
\text { (2011) }\end{array}$ \\
\hline$N H L^{d}$ & & & Insecticide & $\mathrm{OR}=1.67(1.25-2.24)$ & $<0.01$ & $\begin{array}{l}\text { Moderately exposed ( } 2-4 \text { pesti- } \\
\text { cides) vs non-exposed }\end{array}$ & $\begin{array}{l}\text { Hohenadel et al. } \\
\text { (2011) }\end{array}$ \\
\hline$N H L^{d}$ & & & Fungicide & $\mathrm{OR}=1.72(1.07-2.77)$ & 0.04 & $\begin{array}{l}\text { Highly exposed ( } \geq 2 \text { pesticides) vs } \\
\text { non-exposed }\end{array}$ & $\begin{array}{l}\text { Hohenadel et al. } \\
\text { (2011) }\end{array}$ \\
\hline$N H L^{d}$ & & Phenoxy & Herbicide & $\mathrm{OR}=1.78(1.27-2.50)$ & 0.01 & $\begin{array}{l}\text { Highly exposed ( } \geq 2 \text { pesticides) vs } \\
\text { non-exposed }\end{array}$ & $\begin{array}{l}\text { Hohenadel et al. } \\
\text { (2011) }\end{array}$ \\
\hline$N H L^{d}$ & & $\begin{array}{l}\text { Organophos- } \\
\text { phate }\end{array}$ & Insecticide & $\mathrm{OR}=1.69(1.04-2.74)$ & $<0.01$ & $\begin{array}{l}\text { Highly exposed ( } \geq 2 \text { pesticides) vs } \\
\text { non-exposed }\end{array}$ & $\begin{array}{l}\text { Hohenadel et al. } \\
\text { (2011) }\end{array}$ \\
\hline$N H^{d}$ & $\begin{array}{l}\text { Potentially } \\
\text { carcinogenic }\end{array}$ & & & $\mathrm{OR}=1.94(1.17-3.23)$ & 0.01 & $\begin{array}{l}\text { Highly exposed ( } \geq 5 \text { pesticides) vs } \\
\text { non-exposed }\end{array}$ & $\begin{array}{l}\text { Hohenadel et al. } \\
\text { (2011) }\end{array}$ \\
\hline$N H L^{d}$ & DDT & Organochlorine & Insecticide & $\mathrm{RR}=1.7(1.1-2.6)$ & 0.02 & $\begin{array}{l}\text { Highly exposed ( } \geq 56 \text { LD) vs non- } \\
\text { exposed }\end{array}$ & Alavanja et al. (2014) \\
\hline$N H L^{d}$ & Lindane & Organochlorine & Insecticide & $\mathrm{RR}=2.5(1.4-4.4)$ & 0.004 & $\begin{array}{l}\text { Highly exposed ( } \geq 56 \text { LD) vs non- } \\
\text { exposed }\end{array}$ & Alavanja et al. (2014) \\
\hline$N H L^{d}$ & Terbufos & $\begin{array}{l}\text { Organophos- } \\
\text { phate }\end{array}$ & Insecticide & $\mathrm{RR}=1.2(1.0-1.5)$ & & Ever vs never exposure & Alavanja et al. (2014) \\
\hline Ovary & Diazinon & $\begin{array}{l}\text { Organophos- } \\
\text { phate }\end{array}$ & Insecticide & $\mathrm{RR}=1.87(1.02-3.43)$ & & Ever vs never use & Lerro et al. (2015) \\
\hline Pancreatic & EPTC & Thiocarbamate & Herbicide & $\mathrm{OR}=1.8(1.0-3.3)$ & & Ever vs never exposure & $\begin{array}{l}\text { Andreotti et al. } \\
\text { (2009) }\end{array}$ \\
\hline Pancreatic & EPTC & Thiocarbamate & Herbicide & $\mathrm{OR}=2.5(1.1-5.4)$ & 0.01 & $\begin{array}{l}\text { Highly exposed ( } \geq 118 \text { IWLD) vs } \\
\text { non-exposed }\end{array}$ & $\begin{array}{l}\text { Andreotti et al. } \\
\text { (2009) }\end{array}$ \\
\hline Pancreatic & Pendimethalin & Dinitroanilines & Herbicide & $\mathrm{OR}=3.0(1.3-7.2)$ & 0.01 & $\begin{array}{l}\text { Highly exposed ( } \geq 117 \text { IWLD) vs } \\
\text { non-exposed }\end{array}$ & $\begin{array}{l}\text { Andreotti et al. } \\
\text { (2009) }\end{array}$ \\
\hline
\end{tabular}




\begin{tabular}{|c|c|c|c|c|c|c|c|}
\hline Prostate & Butylate & Thiocarbamate & Herbicide & $\mathrm{RR}=1.44(1.04-2.00)$ & 0.03 & $\begin{array}{l}\text { Highly exposed ( } \geq 57 \text { LD) vs } \\
\text { non-exposed }\end{array}$ & Lynch et al. (2009) \\
\hline Prostate & Coumaphos & $\begin{array}{l}\text { Organophos- } \\
\text { phate }\end{array}$ & Insecticide & $R R=1.91(1.23-2.95)$ & 0.004 & Ever vs never use & $\begin{array}{l}\text { Christensen et al. } \\
\text { (2010) }\end{array}$ \\
\hline Prostate & Methyl bromide & Organobromine & Fungicide & $\mathrm{OR}=1.62(1.02-2.59)$ & & Exposed vs non-exposed & Cockburn et al. (2011) \\
\hline Prostate & & Organochlorinef & Insecticide & $\mathrm{OR}=1.64(1.02-2.63)$ & & Exposed vs non-exposed & Cockburn et al. (2011) \\
\hline Prostate & DDT & Organochlorine & Insecticide & $\mathrm{OR}=1.68(1.04-2.70)$ & 0.03 & Highly exposed vs non-exposed & Band et al. (2011) \\
\hline Prostate & Lindane & Organochlorine & Insecticide & $\mathrm{OR}=2.02(1.15-3.55)$ & 0.03 & Highly exposed vs non-exposed & Band et al. (2011) \\
\hline Prostate & $\begin{array}{l}\text { 3,5-dinitro-o- } \\
\text { cresol }\end{array}$ & Organic & Insecticide & $\mathrm{OR}=1.80(1.05-3.08)$ & 0.03 & Highly exposed vs non-exposed & Band et al. (2011) \\
\hline Prostate & $\begin{array}{l}\text { Azinphos- } \\
\text { methyl }\end{array}$ & $\begin{array}{l}\text { Organophos- } \\
\text { phate }\end{array}$ & Insecticide & $\mathrm{OR}=1.88(1.06-3.32)$ & 0.01 & Highly exposed vs non-exposed & Band et al. (2011) \\
\hline Prostate & Carbaryl & Carbamate & Insecticide & $\mathrm{OR}=1.73(1.09-2.74)$ & 0.01 & Highly exposed vs non-exposed & Band et al. (2011) \\
\hline Prostate & Diazinon & $\begin{array}{l}\text { Organophos- } \\
\text { phate }\end{array}$ & Insecticide & $\mathrm{OR}=1.93(1.21-3.08)$ & 0.02 & Highly exposed vs non-exposed & Band et al. (2011) \\
\hline Prostate & Malathion & $\begin{array}{l}\text { Organophos- } \\
\text { phate }\end{array}$ & Insecticide & $\mathrm{OR}=1.49(1.02-2.18)$ & 0.03 & Highly exposed vs non-exposed & Band et al. (2011) \\
\hline Prostate & $2,4-\mathrm{DB}$ & $\begin{array}{l}\text { Chlorinated } \\
\text { phenol }\end{array}$ & Herbicide & $\mathrm{OR}=2.19(1.06-4.50)$ & 0.02 & Highly exposed vs non-exposed & Band et al. (2011) \\
\hline Prostate & MCPA & $\begin{array}{l}\text { Chlorinated } \\
\text { phenol }\end{array}$ & Herbicide & $\mathrm{OR}=2.31(1.09-4.88)$ & 0.02 & Highly exposed vs non-exposed & Band et al. (2011) \\
\hline Prostate & Simazine & Triazine & Herbicide & $\mathrm{OR}=1.89(1.08-3.33)$ & 0.01 & Highly exposed vs non-exposed & Band et al. (2011) \\
\hline Prostate & Copper sulfate & Inorganic & Fungicide & $\mathrm{OR}=1.74(1.04-2.91)$ & 0.05 & Highly exposed vs non-exposed & Band et al. (2011) \\
\hline Prostate & Dichlone & Napthoquinone & Fungicide & $\mathrm{OR}=1.88(1.01-3.52)$ & 0.02 & Highly exposed vs non-exposed & Band et al. (2011) \\
\hline Prostate & Ferbam & Carbamate & Fungicide & $\mathrm{OR}=1.90(1.09-3.30)$ & 0.02 & Highly exposed vs non-exposed & Band et al. (2011) \\
\hline Prostate & Maneb & Dithiocarbamate & Fungicide & $\mathrm{OR}=1.90(1.09-3.30)$ & 0.02 & Highly exposed vs non-exposed & Band et al. (2011) \\
\hline Prostate & Sulfur & & Fungicide & $\mathrm{OR}=1.81(1.12-2.92)$ & 0.02 & Highly exposed vs non-exposed & Band et al. (2011) \\
\hline Prostate & Ziram & Carbamate & Fungicide & $\mathrm{OR}=1.83(1.08-3.10)$ & 0.03 & Highly exposed vs non-exposed & Band et al. (2011) \\
\hline Prostate & Captan & Phentolamine & Fungicide & $\mathrm{OR}=1.76(1.12-2.78)$ & 0.02 & Low exposed vs non-exposed & Band et al. (2011) \\
\hline Prostate & Terbufos & $\begin{array}{l}\text { Organophos- } \\
\text { phate }\end{array}$ & Insecticide & $H R=1.28(1.06-1.55)$ & $>0.05$ & $\begin{array}{l}\text { Moderately exposed } \\
(107<\mid \text { WLD>352) vs non- } \\
\text { exposed }\end{array}$ & Bonner et al. (2010) \\
\hline Prostate & & & $\begin{array}{l}\text { Insecticide } \\
+ \text { fungicide }\end{array}$ & $\mathrm{OR}=2.23(1.15-4.33)$ & & Men exposed vs non-exposed & Sharma et al. (2016) \\
\hline Stomach & Methyl bromide & Organobromine & Fungicide & $R R=3.13(1.25-7.80)$ & 0.02 & $\begin{array}{l}\text { Highly exposed (>765 IWLD) vs } \\
\text { non-exposed }\end{array}$ & Barry et al. (2012) \\
\hline $\begin{array}{l}\text { Soft tissue } \\
\text { sarcoma }\end{array}$ & Aldrin & Organochlorine & Insecticide & $\mathrm{OR}=3.71(1.00-13.76)$ & & Exposed vs non-exposed & Pahwa et al. (2011) \\
\hline $\begin{array}{l}\text { Soft tissue } \\
\text { sarcoma }\end{array}$ & Diazinon & $\begin{array}{l}\text { Organophos- } \\
\text { phate }\end{array}$ & Insecticide & $\mathrm{OR}=3.31(1.78-6.23)$ & & Exposed vs non-exposed & Pahwa et al. (2011) \\
\hline Thyroid & Atrazine & Organic & Herbicide & $R R=4.84(1.31-17.93)$ & 0.08 & $\begin{array}{l}\text { Q4 (>178.5 IWLD) vs Q1( } \leq 20 \\
\text { IWLD) }\end{array}$ & Freeman et al. (2011) \\
\hline Thyroid & Malathion & $\begin{array}{l}\text { Organophos- } \\
\text { phate }\end{array}$ & Insecticide & $R R=2.04(1.14-3.63)$ & & Ever vs never use & Lerro et al. (2015) \\
\hline
\end{tabular}

aAcute lymphoblastic leukemia. ${ }^{b}$ Acute myeloid leukemia. cLymphohematopoietic cancer. dNon-Hodgkin lymphoma. eAccording to the Pesticide Management Education Program (http://pmep.cce.cornell.edu/profiles/index.html). 'Dicofol, dieldrin, dienochlor, endosulfan, heptachlor, lindane, methoxychlor, and toxaphene. $80 R=$ Odd Ratio; $R R=$ Relative Risk; $H R=$ Hazard Ratio. ${ }^{h L D}=$ lifetime days of pesticide use, i.e., the product of years of use of a specific pesticide and the number of days used per year; IWLD= intensity-weighted lifetime days of use, i.e., the product of lifetime days of use and a measure of exposure intensity; T3= third tertile, Q4=fourth quantile. 
Chart 3. Registration status of pesticides positively associated with cancer - European Union, United States, and Brazil

\begin{tabular}{|c|c|c|c|}
\hline \multirow[t]{2}{*}{ Pesticide } & \multicolumn{3}{|c|}{ Registration Status } \\
\hline & European Union ${ }^{a}$ & United States $^{b}$ & Brazilc \\
\hline $2,4,5-T$ & Not Approved & Banned or Severely Restricted & Banned \\
\hline $2,4-D$ & Approved & Banned or Severely Restricted & Approved, but under review \\
\hline $2,4-\mathrm{DB}$ & Approved & Registration Review & Banned \\
\hline 3,5-dinitro-o-cresol & Not registered & Not registered & Not registered \\
\hline Aldrin & Not Approved & Banned or Severely Restricted & Banned \\
\hline Alachlor & Not Approved & Reregistration & Approved \\
\hline Atrazine & Not Approved & Registration Review & Approved \\
\hline Azinphos-methyl & Not Approved & Banned or Severely Restricted & Not approved \\
\hline Bentazon & Approved & Registration Review & Approved \\
\hline Bromoxynil & Approved & Registration Review & Approved \\
\hline Butylate & Not Approved & Registration Review & Banned \\
\hline Captan & Approved & Registration Review & Approved \\
\hline Carbaryl & Not Approved & Registration Review & Approved \\
\hline Carbofuran & Not Approved & Banned or Severely Restricted & Banned \\
\hline Chloramben & Not Approved & Approved & Banned \\
\hline Chlorimuron ethyl & Not Approved & Registration Review & Approved \\
\hline Chlorpyrifos & Approved & Registration Review & Approved \\
\hline Copper sulfate & Approved & Registration Review & Approved \\
\hline Coumaphos & Not Approved & Registration Review & Not registered \\
\hline d-Allethrin & Not Approved & Registration Review & Approved \\
\hline DDT & Not Approved & Banned or Severely Restricted & Banned \\
\hline Diazinon & Not Approved & Registration Review & Approved \\
\hline Dichlone & Not Approved & Approved & Not registered \\
\hline Dichlorprop & Not Approved & Approved & Approved \\
\hline Diclofop-methyl & Approved & Registration Review & Approved \\
\hline d-phenothrin & Not Approved & Registration Review & Approved \\
\hline EPTC & Not Approved & Registration Review & Banned \\
\hline Esbiothrin & Not registered & Registration Review & Approved \\
\hline Ferbam & Not Approved & Reregistration & Not registered \\
\hline Glyphosate & Approved & Registration Review & Approved, but under review \\
\hline Imazaquin & Approved & Registration Review & Approved \\
\hline Imazethapyr & Not Approved & Registration Review & Approved \\
\hline Imiprothrin & Not registered & Registration Review & Approved \\
\hline Lindane & Not Approved & Banned or Severely Restricted & Banned \\
\hline Malathion & Approved & Registration Review & Approved \\
\hline Maneb & Not Approved & Registration Review & Banned \\
\hline Mancozeb & Approved & Reregistration & Approved \\
\hline MCPA & Approved & Registration Review & Approved \\
\hline
\end{tabular}




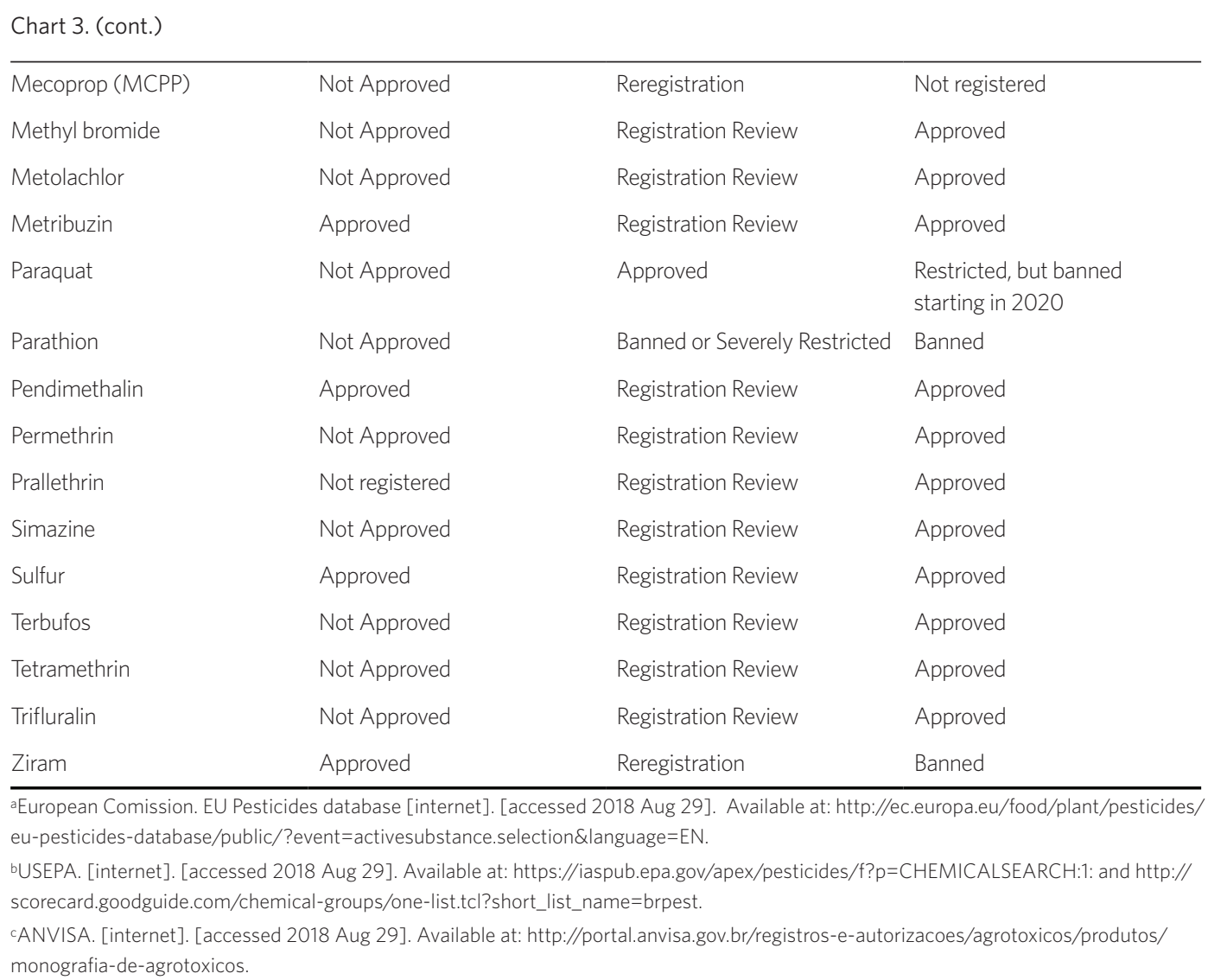

\section{Discussion}

From the 53 pesticides listed in chart 2 relating to at least one type of cancer, most are still being used in the United States (44) and Brazil (34) (chart 3). From this list, only 8 pesticides are currently not approved nor registered, banned or severely restricted in the United States, the European Union, and Brazil: 2,4,5-T, 3,5-dinitro-o-cresol, aldrin, azinphos-methyl, carbofuran, DDT, lindane, and parathion. The pesticides mostly related to cancers fell into the category of the herbicides (24), insecticides (19), and fungicides (9) (chart 2). The most frequent chemical groups associated with cancers included organophosphates, pyrethroids, organochlorines, and thiocarbamates (chart 2).

Results from the Agricultural Health Study (AHS), a prospective cohort of licensed pesticide applicators from Iowa and North Carolina (USA), indicated that the highest levels of EPTC $^{9}$ and butylate ${ }^{10}$ lifetime exposure days (LD) were associated to all cancers. Additionally, moderate and high exposures to terbufos also increased overall cancer hazard ratio"1. On the other hand, some cohort studies investigated specific pesticides such as chlorothalonil ${ }^{12}$, $\operatorname{captan}^{\mathbf{1 3}}$, malathion ${ }^{\mathbf{1 4}}$, and dichlor$\operatorname{vos}^{15}$, although not finding any association with cancer.

To reside near crops was reported to increase cancer risk in children younger than $14^{16}$ or 15 years old ${ }^{17}$. However, another study ${ }^{\mathbf{1 8}}$ evaluated several types of childhood cancers and was not able to find any significant association with residence near agricultural fields.

Being a farmer also significantly increased overall cancer risk (OR=1.459, 95\% CI: 1.2291.731) when compared to non-farmers of the 
same gender and age range ${ }^{19}$. Lemarchand et al. ${ }^{20}$ also observed significantly higher overall cancer risk among male farm workers, measured by the Standardized Incidence Ratio (SIR) of 1.07, 95\% CI: 1.03-1.12.

Several studies analyzed neoplasms of the hematopoietic and lymphoid tissues (LHC) and found significantly increased risk in people living in a farm ${ }^{21}$ or near crops ${ }^{16}$ exposed to pesticides ${ }^{\mathbf{2 2 - 2 4}}$, butylate herbicide ${ }^{\mathbf{1 0}}$, metribuzin herbicide ${ }^{22}$, or terbufos insecticide ${ }^{11}$.

Leukemia primarily affects children. Several studies found association between different types of childhood leukemia and pesticide exposure ${ }^{25-27}$. Residing near certain crops $^{28}$, or in counties of high level of agricultural activity ${ }^{17}$, was also found to significantly increase the risk of childhood cancer. Although Malagoli et al. ${ }^{29}$ could not find statistically significant results, they suggested that childhood leukemia risk increased when the child resides near arable crops. Children who were born in rural areas (RR=1.43, 95\% CI: 1.09-1.86, p-trend $=0.003$ ) or in counties with the highest farming index ( $\mathrm{RR}=1.33$, 95\% CI: 1.04-1.69) or pesticide exposure index ( $R R=1.30,95 \%$ CI: 1.02-1.66) faced significantly higher risk to die from leukemia ${ }^{30}$. In adults, increased leukemia risk was significantly associated with exposure to EPTC herbicide 9 and terbufos insecticide ${ }^{11}$. Other risk factors related to a farm life such as living on a farm, planting crops, raising livestock or animals, working as farm workers or in the agricultural industry, and exposures to insecticides or fertilizers ${ }^{31}$.

Hodgkin Lymphoma (HL) in males of 19 years of age or older was significantly associated with exposure to the organophosphate insecticide chlorpyrifos ${ }^{32}$ and the herbicide dichlorprop ${ }^{33}$. Hodgkin's disease and NonHodgkin Lymphoma (NHL) were significantly reduced in districts with low pesticides exposure compared to those with high exposure ${ }^{34}$.

Non-Hodgkin lymphoma risk factors include: being an agricultural worker ${ }^{35-37}$ or a farmer 35,38,39; living in a farm or in communities between 1,000 and 10,000 people ${ }^{39}$; being exposed to pesticides ${ }^{39}$, potentially carcinogenic pesticides ${ }^{40}$, herbicides ${ }^{35,38,40}$,

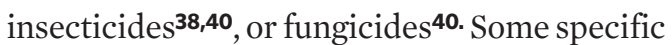
insecticides such as DDT ${ }^{41}$, lindane ${ }^{41}$, and terbufos $^{\mathbf{1 1}, \mathbf{4 1}}$, as well as some specific herbicides such as butylate ${ }^{10}$ and paraquat ${ }^{42}$, were also associated with higher risk of NHL.

Multiple myeloma was associated to six specific types of pesticides. Otherwise, results were contradictory for captan fungicide and carbaryl insecticide. While three case-control studies ${ }^{\mathbf{4 3 - 4 5}}$ showed that these pesticides increased MM risk, one cohort study ${ }^{\mathbf{4 1}}$ could not find significant associations. Different results also appeared for DDT and lindane insecticides. Presutti et al. ${ }^{45}$ found DDT to be linked to MM, but could not trace significant correlation between lindane and MM. Conversely, Kachuri et al. ${ }^{\mathbf{4 3}}$ and Pahwa et al. ${ }^{\mathbf{4 4}}$ found DDT not to be linked to MM, while lindane showed a significant association. Two cohort studies investigated permethrin insecticide ${ }^{41,46}$, and other two case-control studies $\mathbf{4 3 , 4 4}^{\mathbf{4}}$ investigated mecoprop herbicide, and they all found significant high MM risk. Consistency was also seen among the four studies about not finding significant associations between malathion and MM ${ }^{41,43-45}$. Furthermore, increased risk of MM was seen among men who reported the use of fungicides, pesticides classified as probably carcinogenic or higher, using at least one carbamate pesticide, one phenoxy herbicide, and 3 organochlorines ${ }^{43}$. Occasional, although intense, use of pesticides or herbicides by men also caused a significant MM excess risk (RR=1.20, 95\% CI: 1.07-1.34) ${ }^{47}$. Female crop farmers ${ }^{37}$, as well as female and male pesticide users ${ }^{\mathbf{4 8}}$, suffered higher incidences of MM. Similarly, a study ${ }^{\mathbf{2 0}}$ observed higher risks among males and females who work in farms and among male farm owners (SIR=1.59 95\% CI: 1.29-1.95) and male users of pesticides on crops (SIR=1.49, 95\% CI: 1.19-1.84).

Although the main risk factors, i.e., age, black race, family history, related to prostate neoplasm are already identified, this integrative review revealed that exposure 
to butylate ${ }^{10}$, methyl bromide ${ }^{49}$, a group of organochlorine insecticide ${ }^{\mathbf{4 9}}$, and terbufos ${ }^{\mathbf{1 1}}$ were found to increase the risk. High exposure to the (i) insecticides DDT, lindane, 3,5-dinitro-cresol, azinphos-methyl, carbaryl, diazinon, malathion, (ii) herbicides 2,4-DB, MCPA, simazine, and (iii) fungicides copper sulfate, dichlone, ferbam, maneb, sulfur, ziram significantly increased prostate cancer risk in males ${ }^{\mathbf{5 0}, 51}$. Prostate cancer risk was higher among male agricultural workers ${ }^{20,37}$ and men exposed to coumaphos who reported a family history of that cancer ${ }^{52}$.

Primary testicular tumors are the most common solid malignant tumor in men aged 20 to 34 years in the United States ${ }^{53}$ and its cause is still unknown, although a study has evidenced that its incidence was significantly higher among male pesticide users (SIR=1.26, 95\% CI: $1.04-1.53)^{\mathbf{4 8}}$.

Among malignant neoplasms of digestive organs, the herbicides EPTC and pendimethalin were associated with pancreatic cancer among pesticide applicators and their spouses ${ }^{37,54}$. Stomach cancer risk significantly increased with exposure to methyl bromide ${ }^{55}$ and in districts with greater pesticide use ${ }^{34}$. Colorectal cancer risk was significantly higher among farmers (OR=1.529, 95\% CI: 1.011-2.314) $)^{\mathbf{1 9}}$, those exposed to pesticide (OR=2.6, 95\% CI: 1.1-5.9), and those primarily sourcing food directly from farms (OR=4.6, 95\% CI: 1.5-14.6) ${ }^{56}$. A higher prevalence of colon cancer was also observed among male pesticide applicators exposed to EPTC ${ }^{9}$, trifluralin ${ }^{57}$, carbofuran, metolachlor, and alachlor ${ }^{58}$. Esophagus cancer deaths were, in general, significantly higher (OR=1.38, 95\% CI: 1.26-1.51) among agricultural than among non-agricultural workers in the south region of Brazil, an area with intense pesticide use ${ }^{59}$. The Hepatocellular Carcinoma (HCC) can be affected by several factors, and pesticide exposure may contribute to non-B and non-C HCC in areas with high level of agricultural activity 17,34,60-62. In contrast, Jeephet et al. ${ }^{63}$ were not able to find statistically significant association between pesticide use and cholangio carcinoma.

Central nervous system tumors increased among farmers (HR= 1.73, 95\% CI: 1.01-2.94) ${ }^{64}$, pesticide applicators (HR=1.96; 95\% CI: 1.11$3.47)^{64}$, and children living in countries with high level of agricultural activity (OR=1.3, 95\% CI: 1.1-1.4) $)^{17}$. Brain cancer prevalence ${ }^{34}$ and its mortality ${ }^{65,66}$ showed significantly higher rates in districts with greater pesticide use. Glioma was associated with never changing clothes $(\mathrm{OR}=2.84$, 95\% CI: 1.04-7.78) or never washing face and hands (OR=3.08, 95\% CI: 1.78-5.34) immediately after applying pesticides ${ }^{67}$. Controversially, a study investigating pesticide applicators did not find any positive association between glioma and farm pesticide use ${ }^{68}$.

As for malignant neoplasms of female genital organs, a study ${ }^{69}$ investigated risk factors for cervical cancer and could not find any association with insecticides. The result was anticipated, once most cervical cancer cases are caused by the human papillomavirus, a well-known risk factor. Ashley-Martin et al. ${ }^{70}$ did not find significant associations between breast cancer and fungicide exposure. However, Salerno et al. ${ }^{19}$ observed that farmers were at significantly higher risk for breast cancer (OR=1.720, 95\% CI: 1.039-2.846), and Lerro et al..$^{71}$ found organophosphate insecticides to be associated with breast tumor and diazinon to significantly increase the risk of ovarian cancer.

Among malignant neoplasms of urinary tract, bladder cancer revealed to be the most common type associated with pesticides. The prevalence was significantly higher in districts with greater pesticide use ${ }^{34}$. Any use of imazethapyr, imazaquin, bentazon, bromoxynil, chloramben, and diclofop-methyl herbicides increased the risk of bladder cancer, as did the insecticide DDT solely ${ }^{\mathbf{2}, 73}$. In contrast, a study ${ }^{\mathbf{7 4}}$ investigating risk factors for bladder cancer among farm workers could not find any significant increasing risk for pesticide exposure, whilst significant high risk was observed 
among field-grown vegetable workers. Renal tumors were associated with living in counties with high level of agricultural activity (OR=2.1, 95\% CI: 1.7-2.6) ${ }^{\mathbf{1 7}}$.

Lung cancer is the primary contributor of malignant neoplasms of respiratory and intrathoracic organs. After controlling for several factors including smoking, which is the most common risk factor, lung cancer among pesticide applicators from the AHS cohort was significantly associated to high exposure to the organophosphate insecticide diazinon ( $\mathrm{RR}=1.60$, 95\% CI: 1.11-2.31) ${ }^{75}$. The highest quartile of use of the herbicide chlorimuron ethyl showed high risk of lung cancer ${ }^{76}$. Significantly higher prevalence was also observed in districts with greater pesticide use ${ }^{34}$.

Cutaneous melanoma incidence among pesticide applicators was significantly increased by the exposure to parathion and carbaryl insecticides and maneb/mancozeb fungicide after adjusting for risk factors ${ }^{77,78}$. A higher risk for skin melanoma $(\mathrm{SIR}=1.30,95 \% \mathrm{CI}$ : 1.00-1.66) was observed among female farm workers ${ }^{20}$. Additionally, an increased melanoma hazard ratio among male agricultural workers and female crop farmers was also identified ${ }^{37}$. A study investigated uveal melanoma but could not find positive associations with activities of farming, pesticide application, or pesticide mixing79.

Soft Tissue Sarcoma (STS) was significantly associated to also exposure to aldrin and diazinon among men aged 19 years or older ${ }^{80}$ as to with high level of agricultural activity (OR=1.7, 95\% CI: 1.4-2.0) ${ }^{17}$. Among British women, it was observed that pesticide users died more often from STS than the national population ${ }^{48}$. Malignant bone tumors were associated to living in counties with high level of agricultural activity (OR=2.3, 95\% CI: 1.8-2.9) ${ }^{17}$.

Head and neck cancer was reported among men and women residing in rural areas ${ }^{81}$. Thyroid cancer risk increased with malathion ${ }^{71}$ and atrazine exposure ${ }^{82}$. Lip cancer risk was significantly higher among male agricultural workers (HR=
2.14, 95\% CI: 1.70-2.70) $)^{37}$ and male farm workers (SIR=2.87, 95\% CI: 1.61-4.74) ${ }^{\mathbf{2 0}}$.

Myelodysplastic Syndromes (MDS) were significantly associated to ever exposure to pesticides (OR=2.47, 95\% CI: 1.44-4.24), insecticides (OR=3.34, 95\% CI: 1.62-6.90) and herbicides (OR $=2.27,95 \%$ CI: $1.14-4.51)$, but not to fungicides ${ }^{83}$. Paraquat was the only specific pesticide to positively and significantly associate with MDS (OR= 4.90, 95\% CI: 1.05-22.75).

The choice for an integrative review may be considered one of the strengths of this study, since it is the only approach that allows for combining results of different methodologies. This study has the potential to enable for the diversity in primary research to be summarized and to become an instrument also for medical professionals that deal with cancers as for decision-makers responsible for making the public policies, once risks to populations were identified.

As for its limitations, this study focused on a very wide topic that encompassed all kinds of pesticides and cancers, which may have led to the loss of specific details. Second, it was only able to analyze the registration status of pesticides in the United States, Brazil, and the European Union, since most of the papers retrieved from the Medline/PubMed database belonged to those places. It would certain be beneficial to further add other countries to the comparison. It is important to note that half of the studies retrieved were carried out in the USA, being 25 published by AHS researchers. Epidemiologic evidence outside the AHS cohort remains limited as far as associations observed for specific pesticides and cancer types are concerned. Third and last, this study did not discuss potential mechanisms of action of pesticides that could have improved the study.

\section{Conclusions}

This integrative literature review showed that the risk of several cancer types increased 
significantly with exposure to several types of pesticides, most of which are still in use in the United States and Brazil. Although a few studies presented contradictory results, being a farmer or living near crops or high agricultural areas have also been used as a proxy for pesticide exposure and significantly associated with higher cancer risk.

In general, the literature is well illustrated in the case of prostate cancer, NHL, leukemia, multiple myeloma, bladder and colon cancers. Studies that further investigate the relationship between pesticide and neoplasms of testis, breast, esophagus, kidney, thyroid, lip, head/neck, and bone are recommended. It is hoped that this study can be used as a reference material and will contribute to future research regarding pesticide exposure and cancer incidence.

\section{Collaborators}

Pluth TB (0000-0002-5851-9476)* made substantial contribution to the conception, design, drafting of the work, and to the analysis and interpretation of data. Zanini LAG (0000-0002-3849-6211)* contributed to the critical review of the content, and assisted in data interpretation and drafting of the work. Battisti IDE (0000-0001-9740-4199)* made substantial contribution to the conception and design of the work. All authors approved the final version to be published.

\section{References}

1. World Health Organization. Pesticides [internet]. Genebra: WHO; 2019 [accessed 2017 Sept 10]. Available at: http://www.who.int/topics/pesticides/en/.

2. Garcia EG, Alves Filho JP. Aspectos de prevenção e controle de acidentes no trabalho com agrotóxicos. São Paulo: Fundacentro; 2005.

3. Peres F. Saúde, trabalho e ambiente no meio rural brasileiro. Ciênc. Saúde Colet. 2009; 14:1995-2004.

4. Brito PF, Gomide M, Magalhães V, et al. Familiar agriculture and pesticide exposure : brief considerations. Cad. Saúde Colet. 2005; 13(4):887-900

5. Pacheco MEL, Guimarães MK, Silva LR. Mesa de controvérsias sobre o impacto dos agrotóxicos na soberania e segurança alimentar e nutricional e no di- reito humano a alimentação adequada. Brasília, DF: CONSEA; 2014.

6. Miller GT. Biodiversity: sustaining soils and producing food. In: Miller GT. Sustaining the Earth. 6. ed. Pacific Grove, California: Thompson Learning, Inc.; 2004. p. 211-216.

7. Weichenthal S, Moase C, Chan P. A review of pesticide exposure and cancer incidence in the agricultural health study cohort. Environ. Health Perspect. 2010; 118(8):1117-1125.

8. Ferlay J, Soerjomataram I, Ervik M, et al. GLOBOCAN 2012 vl.o, Cancer Incidence and Mortality Worldwide: IARC CancerBase No. 11 [internet]. Lyon, France: International Agency for Research on Cancer; 2013. [accessed 2017 Feb 27]. Available at: http://globocan.iarc.fr. 
9. Van Bemmel DM, Visvanathan K, Beane Freeman LE, et al. S-ethyl-N,N-dipropylthiocarbamate exposure and cancer incidence among male pesticide applicators in the agricultural health study: A prospective cohort. Environ Health Perspect. 2008; 116(11):1541-1546.

10. Lynch SM, Mahajan R, Beane Freeman LE, et al. Cancer incidence among pesticide applicators exposed to butylate in the Agricultural Health Study (AHS). Environ Res. 2009; 109(7):860-868.

11. Bonner MR, Williams BA, Rusiecki JA, et al. Occupational Exposure to Terbufos and the Incidence of Cancer in the Agricultural Health Study. Cancer Causes Control. 2010; 21(6):871-877.

12. Mozzachio AM, Rusiecki JA, Hoppin JA, et al. Chlorothalonil exposure and cancer incidence among pesticide applicator participants in the agricultural health study. Environ Res. 2008; 108(3):400-403.

13. Greenburg DL, Rusiecki J, Koutros S, et al. Cancer incidence among pesticide applicators exposed to captan in the Agricultural Health Study. Cancer Causes Control. 2008; 19(10):1401-1407.

14. Bonner MR, Coble J, Blair A, et al. Malathion exposure and the incidence of cancer in the agricultural health study. Am J Epidemiol. 2007; 166(9):1023-1034.

15. Koutros S, Mahajan R, Zheng T, et al. Dichlorvos Exposure and Human Cancer Risk: Results from the Agricultural Health Study. Cancer Causes Control. 2008; 19(1):59-65.

16. Gómez-Barroso D, García-Pérez J, López-Abente G, et al. Agricultural crop exposure and risk of childhood cancer: new findings from a case-control study in Spain. Int J Health Geogr. 2016; 15(1):18.

17. Carozza SE, Li B, Elgethun K, et al. Risk of childhood cancers associated with residence in agriculturally intense areas in the United States. Environ Health Perspect. 2008; 116(4):559-565.

18. Carozza SE, Li B, Wang Q, et al. Agricultural pesticides and risk of childhood cancers. Int J Hyg Envi- ron Health. 2009; 212(2):186-195.

19. Salerno C, Carcagnì A, Sacco S, et al. An Italian population-based case-control study on the association between farming and cancer: are pesticides a plausible risk factor? Arch Environ Occup Heal. 2016; 71(3):147-156.

20. Lemarchand C, Tual S, Levêque-Morlais N, et al. Cancer incidence in the AGRICAN cohort study (20052011). Cancer Epidemiol. 2017; 49:175-185.

21. Jones RR, DellaValle CT, Flory AR, et al. Accuracy of residential geocoding in the Agricultural Health Study. Int. j. health geogr. 2014; 13(37):1-9.

22. Delancey JOL, Alavanja MCR, Coble J, et al. Occupational Exposure to Metribuzin and the Incidence of Cancer in the Agricultural Health Study. Ann. epidemiol. 2009; 19(6):388-395.

23. Kokouva M, Bitsolas N, Hadjigeorgiou GM, et al. Pesticide exposure and lymphohaematopoietic cancers: a case-control study in an agricultural region (Larissa, Thessaly, Greece). BMC public health. 2011; 11(1):1-5.

24. Schinasi LH, De Roos AJ, Ray RM, et al. Insecticide exposure and farm history in relation to risk of lymphomas and leukemias in the Women's Health Initiative observational study cohort. Ann. epidemiol. 2015; 25(11):803-810.

25. Rull RP, Gunier R, Von Behren J, et al. Residential proximity to agricultural pesticide applications and childhood acute lymphoblastic leukemia. Environ Res. 2009; 109(7):891-899.

26. Ferreira JD, Couto AC, Pombo-de-Oliveira MS, et al. In utero pesticide exposure and leukemia in Brazilian children $<2$ years of age. Environ. health perspect. $2013 ; 121(2): 269-275$

27. Kumar A, Vashist M, Rathee R. Maternal factors and risk of childhood leukemia. Asian pac. j. cancer prev. 2014; 15(2):781-784.

28. Booth BJ, Ward MH, Turyk ME, et al. Agricultural 
crop density and risk of childhood cancer in the midwestern United States: an ecologic study. Environ Heal. 2015; 14(82):1-11.

29. Malagoli C, Costanzini S, Heck JE, et al. Passive exposure to agricultural pesticides and risk of childhood leukemia in an Italian community. Int. j. hyg. environ. health. 2016; 219(8):742-748.

30. Cha ES, Hwang S, Lee WJ. Childhood leukemia mortality and farming exposure in South Korea: A national population-based birth cohort study. Cancer epidemiol. 2014; 38(4):401-407.

31. Wong O, Harris F, Yiying W, et al. A hospital-based case-control study of acute myeloid leukemia in Shanghai: Analysis of personal characteristics, lifestyle and environmental risk factors by subtypes of the WHO classification. Regul. Toxiol. pharmacol. 2009; 55(3):340-352.

32. Karunanayake CP, Spinelli JJ, McLaughlin JR, et al. Hodgkin Lymphoma and Pesticides Exposure in Men: A Canadian Case-Control Study. J Agromedicine. 2012; 17(1):30-39.

33. Pahwa P, Karunanayake CP, Spinelli JJ, et al. Ethnicity and incidence of Hodgkin lymphoma in Canadian population. BMC cancer. 2009; 9(141):1-9.

34. Parrón T, Requena M, Hernández AF, et al. Environmental exposure to pesticides and cancer risk in multiple human organ systems. Toxicol. lett. 2014; 230(2):157-165.

35. Richardson DB, Terschüren C, Hoffmann W. Occupational Risk Factors for Non-Hodgkin's Lymphoma : A Population-Based Case - Control Study in Northern Germany. Am J Ind Med. 2008; 51:258-268.

36. Boccolini PMM, Boccolini CS, Chrisman JR, et al. Non-Hodgkin lymphoma among Brazilian agricultural workers: A death certificate case-control study. Environ Occup Heal. 2016; 72(3):139-144.

37. Kachuri L, Harris MA, MacLeod JS, et al. Cancer risks in a population-based study of 70,570 agricul- tural workers: results from the Canadian census health and Environment cohort (CanCHEC). BMC cancer. 2017; 17(343):1-15

38. Wong O, Harris F, Armstrong TW, et al. A hospital-based case-control study of non-Hodgkin lymphoid neoplasms in Shanghai: Analysis of environmental and occupational risk factors by subtypes of the WHO classification. Chem. Boil. interact. 2010; 184(1-2):129-146

39. Karunanayake CP, Dosman JA, Pahwa P. Non-hodgkin's lymphoma and work in agriculture: Results of a two case-control studies in Saskatchewan, Canada. Indian j. occup. environ. med. 2013; 17(3):114-121.

40. Hohenadel K, Harris SA, McLaughlin JR, et al. Exposure to multiple pesticides and risk of non-Hodgkin lymphoma in men from six Canadian provinces. Int. j. environ. res. public health. 2011; 8(6):2320-2330.

41. Alavanja MCR, Hofmann JN, Lynch CF, et al. Non-Hodgkin lymphoma risk and insecticide, fungicide and fumigant use in the agricultural health study. PLoS One. 2014; 9(10):1-17.

42. Park SK, Kang D, Beane-freeman L, et al. Cancer incidence among paraquat-exposed pesticide applicators in the Agricultural Health Study. Int J Occup Env Heal. 2009; 15(3):274-281.

43. Kachuri L, Demers PA, Blair A, et al. Multiple pesticide exposures and the risk of multiple myeloma in Canadian men. Int. j. cancer. 2013; 133(8):1846-1858.

44. Pahwa P, Karunanayake CP, Dosman JA, et al. Multiple Myeloma and Exposure to Pesticides: A Canadian Case-Control Study. J Agromedicine. 2012; 17(1):40-50.

45. Presutti R, Harris SA, Kachuri L, et al. Pesticide exposures and the risk of multiple myeloma in men: an analysis of the North American Pooled Project (NAPP). Int. j. cancer. 2016; 139(8):1703-1714.

46. Rusiecki JA, Patel R, Koutros S, et al. Cancer incidence among pesticide applicators exposed to permethrin in the Agricultural Health Study. Environ. 
health perspect. 2009; 117(4):581-586.

47. Lope V, Pérez-Gómez B, Aragonés N, et al. Occupation, exposure to chemicals, sensitizing agents, and risk of multiple myeloma in Sweden. Cancer epidemiol. biomark. prev. 2008; 17(11):3123-3127.

48. Frost G, Brown T, Harding AH. Mortality and cancer incidence among British agricultural pesticide users. Occup Med (Lond). 2011; 61(5):303-310.

49. Cockburn M, Mills P, Zhang X, et al. Prostate cancer and ambient pesticide exposure in agriculturally intensive areas in California. Am j. epidemiol. 2011; 173(11):1280-1288.

50. Band PR, Abanto Z, Bert J, et al. Prostate cancer risk and exposure to pesticides in British Columbia Farmers. Prostate. 2011; 71(2):168-183.

51. Sharma M, Lawson JA, Kanthan R, et al. Factors Associated With the Prevalence of Prostate Cancer in Rural Saskatchewan: The Saskatchewan Rural Health Study. J Rural Heal. 2016; 32(2):125-135.

52. Christensen CH, Platz EA, Andreotti G, et al. Coumaphos exposure and incident cancer among male participants in the Agricultural Health Study (AHS). Environ. health perspect. 2010; 118(1):92-96.

53. United States. National Cancer Institute. Cancer Stat Facts: Testicular Cancer [internet].Maryland: National Cancer Institute; 2019. [accessed 2018 Dec 30]. Available at: https://seer.cancer.gov/statfacts/html/ testis.html.

54. Andreotti G, Freeman LEB, Hou L, et al. Agricultural Pesticide Use and Pancreatic Cancer Risk in the Agricultural Health Study Cohort Gabriella. Int. j. cancer. 2009; 124(10):2495-2500.

55. Barry KH, Koutros S, Lubin JH, et al. Methyl bromide exposure and cancer risk in the Agricultural Health Study. Cancer causes control. 2012; 23(6):807-818.

56. Lo A-C, Soliman AS, Khaled HM, et al. Lifestyle, occupational, and reproductive factors and risk of colorec- tar cancer. Dis. colon rectum. 2010; 53(5):830-837.

57. Kang D, Park SK, Beane-Freeman L, et al. Cancer incidence among pesticide applicators exposed to trifluralin in the Agricultural Health Study. Environ. res. 2008; 107(2):271-276.

58. Andreotti G, Hou L, Freeman LEB, et al. Body Mass Index, Agricultural Pesticide Use, and Cancer Incidence in the Agricultural Health Study Cohort. Cancer causes control. 2010; 21(11):1759-1775.

59. Meyer A, Alexandre PCB, Chrisman JR, et al. Esophageal cancer among Brazilian agricultural workers: Case-control study based on death certificates. Int. j. hyg. environ. health. 2011; 214(2):151-155.

60. Azm ARAE, Yousef M, Mansour N, et al. New insights on non-B non-C hepatocellular carcinoma in mid Delta Region, Egypt. J gastrointest cancer. 2014; 45(3):276-283.

61. VoPham T, Brooks MM, Yuan JM, et al. Pesticide exposure and hepatocellular carcinoma risk: A case-control study using a geographic information system (GIS) to link SEER-Medicare and California pesticide data. Environ. res. 2015; 143:68-82.

62. Silver SR, Bertke SJ, Hines CJ, et al. Cancer incidence and metolachlor use in the Agricultural Health Study: An update. Int. j. cancer. 2015; 137(11):2630-2643.

63. Jeephet K, Kamsa-Ard S, Bhudhisawasdi V, et al. Association between pesticide use and cholangiocarcinoma. Asian pac. J. cancer prev. 2016; 17(8):3977-3980.

64. Piel C, Pouchieu C, Tual S, et al. Central nervous system tumors and agricultural exposures in the prospective cohort AGRICAN. Int. j. cancer. 2017; 141(9):1771-1782.

65. Miranda Filho AL, Monteiro GTR, Meyer A. Brain cancer mortality among farm workers of the State of Rio de Janeiro, Brazil: A population-based case-control study, 1996-2005. Int. j. hyg. environ. health. $2012 ; 215(5): 496-501$.

66. Miranda Filho AL, Koifman RJ, Koifman S, et al. Brain 
cancer mortality in an agricultural and a metropolitan region of Rio de Janeiro, Brazil: a population-based, age-period-cohort study, 1996-2010. BMC cancer. 2014; 14(320):1-9.

67. Ruder AM, Carreón T, Butler MA, et al. Exposure to farm crops, livestock, and farm tasks and risk of glioma. Am. j. epidemiol. 2009; 169(12):1479-1491.

68. Yiin JH, Ruder AM, Stewart PA, et al. The upper midwest health study: a case-control study of pesticide applicators and risk of glioma. Environ Heal. 2012; 11(39):1-13.

69. Zhang B, Zhou AF, Zhu C-C, et al. Risk Factors for Cervical Cancer in Rural Areas of Wuhan China: a Matched Case-control Study. Asian pac. j. cancer prev. 2013; 14(12):7595-7600.

70. Ashley-Martin J, Vanleeuwen J, Cribb A. Breast cancer risk, fungicide exposure and cyplal $* 2$ a gene-environment interactions in a province-wide case control study in prince edward island, Canada. Int. j. environ. res. public health. 2012; 9(5):1846-1858.

71. Lerro CC, Koutros S, Andreotti G, et al. Organophosphate insecticide use and cancer incidence among spouses of pesticide applicators in the Agricultural Health Study. Occup Environ Med. 2015; 72(10):736-744.

72. Koutros S, Lynch CF, Ma X, et al. Aromatic amine pesticide use and human cancer risk: results from the U.S. Agricultural Health Study. Int J Cancer. 2009; 124(5):1206-1212.

73. Koutros S, Silverman DT, Alavanja MCR, et al. Occupational exposure to pesticides and bladder cancer risk. Int J Epidemiol. 2016; 45(3):792-805.

74. Boulanger M, Tual S, Lemarchand C, et al. Agricultural exposure and risk of bladder cancer in the AGRIculture and CANcer cohort. Int Arch Occup Environ Heal. 2017; 90(2):169-178.

75. Jones RR, Barone-Adesi F, Koutros S, et al. Inciden- ce of solid tumours among pesticide applicators exposed to the organophosphate insecticide diazinon in the Agricultural Health Study: an updated analysis. Occup. Environ. med. 2015; 72(7):1-18.

76. Bonner MR, Freeman LEB, Hoppin JA, et al. Occupational Exposure to Pesticides and the Incidence of Lung Cancer in the Agricultural Health Study. Environ. health perspect. 2017; 125(4):544-551.

77. Dennis LK, Lynch CF, Sandler DP, et al. Pesticide use and cutaneous melanoma in pesticide applicators in the agricultural heath study. Environ health perspect. 2010; 118(6):812-817.

78. Mahajan R, Blair A, Coble J, et al. Carbaryl exposure and incident cancer in the Agricultural Health Study. Int j. cancer. 2007; 121(8):1799-1805.

79. Behrens T, Lynge E, Cree I, et al. Pesticide exposure in farming and forestry and the risk of uveal melanoma. Cancer causes control. 2012; 23(1):141-151.

80. Pahwa P, Karunanayake CP, Dosman JA, et al. Soft-Tissue Sarcoma and Pesticides Exposure in Men. J. occup environ. med. 2011; 53(11):1279-1286.

81. Govett G, Genuis SJ, Govett HE, et al. Chlorinated pesticides and cancer of the head and neck. Eur. j. cancer prev. 2011; 20(4):320-325.

82. Freeman LEB, Rusiecki JA, Hoppin JA, et al. Atrazine and cancer incidence among pesticide applicators in the Agricultural Health Study (1994-2007). Environ health perspect. 2011; 119(9):1253-1259.

83. Avgerinou C, Giannezi I, Theodoropoulou S, et al. Occupational, dietary, and other risk factors for myelodysplastic syndromes in Western Greece. Hematology. 2017; 22(7):419-429.

Received on 02/03/2019

Approved on 04/10/2019

Conflict of interest: non-existent

Financial support: non-existent 